\title{
Post-ERCP infection and its epidemiological and clinical characteristics in a large Chinese tertiary hospital: a 4-year surveillance study
}

\author{
Mingmei Du+ ${ }^{1+}$, Jijiang Suo ${ }^{1+}$, Bowei Liu ${ }^{1}$, Yubin Xing ${ }^{1}$, Liangan Chen ${ }^{2^{*}}$ and Yunxi Liu ${ }^{1 *}$
}

\begin{abstract}
Background: Endoscopic retrograde cholangiopancreatography (ERCP) is widely performed as a treatment for biliary and pancreatic illness in China; however, there are few data available regarding post-ERCP infections. This study aimed to describe the overall incidence of post-ERCP infections and the epidemiological characteristics of infected patients in a large tertiary-care hospital in China.

Methods: Real-time surveillance was performed from 2012 through 2015 to identify all healthcare-associated infections (HAls) that occurred after ERCP, using an automatic system. All HAls (e.g., cholangtitis, bacteremia) were identified by infection control practitioners and doctors. Inpatient data were automatically collected by the surveillance system.

Results: A total of 1743 ERCP operations were included in the study, among these, 132 (7.57\%) HAls were identified. ERCP postoperative infections occurred following different surgical procedures, with infection rates ranging from 3.58 to $13.51 \%$. The most prevalent HAI was biliary tract infection (4.02\%), followed by transient bacteremia (1.14\%). Overall, 62 cases of bacteremia occurred following ERCP surgery and 34 (54.84\%) cases occurred on the day of the operation or 1-day post-surgery. The most prevalent isolates detected during bacteremia were Enterococcus faecium (12/58) and Escherichia coli (11/58). A large proportion (72.73\%) of the E. coli isolates and all of the E. faecium isolates were resistant to ciprofloxacin. In addition, only $37.50 \%$ of the E. coli isolates were susceptible to ceftriaxone.

Conclusions: The high incidence of post-ERCP infection and the prevalence of drug resistance suggests that employing second generation cephalosporin or ceftriaxone as the antibiotic of choice for prophylaxis before ERCP, as recommended by the Chinese clinical application of antibacterial drugs guidelines, may not be effective.
\end{abstract}

Keywords: Endoscopic retrograde cholangiopancreatography, healthcare-acquired infections, epidemiology, cholangtitis, bacteremia, antimicrobial resistance, China

\section{Background}

China has a large number of patients with biliary or pancreatic diseases, especially bile duct stones. Currently, endoscopic retrograde cholangiopancreatography (ERCP) plays a major role in the treatment of biliary and pancreatic diseases. The use of ERCP has increased considerably in China in recent years. From

\footnotetext{
* Correspondence: chenliangan301@163.com; liuyunxi301@qq.com ${ }^{\dagger}$ Equal contributors

²Department of Respiratory Medicine, Chinese PLA General Hospital, Fuxing Road No. 28, Beijing 100853, China

${ }^{1}$ Department of Infection Management and Disease Control, Chinese PLA General Hospital, Fuxing Road No. 28, Beijing 100853, China
}

2006 to 2012, the total of ERCP surgeries performed increased from 63,787 to 195,643 in China, of which more than 95\% were therapeutic [1]. Post-ERCP infections have been studied extensively in the USA and European countries, for example, surveillance of post-ERCP bacteremia showed infection rates of 5\% in England [2] and $27.8 \%$ in the USA [3]. However, another retrospective observational study carried out in the USA over an 11-year period indicated that the post-ERCP infection rate was as low as $0.28 \%$ [4].

The variations in surveillance results for post-ERCP infections mainly result from different case definitions, 
varied surveillance durations and the different data collection methods used in previous studies [5]. To date, few studies have been conducted in China. One multicenter cross-sectional questionnaire study that aimed to determine the status of ERCP services in China, reported a cholangitis prevalence of $0.66 \%$ post-ERCP [1]. More research on understanding the incidence of adverse events following ERCP services is required in China.

This study aimed to describe the overall incidence of post-ERCP infection and characterize the infected patients after different ERCP operations. In addition, to improve our knowledge regarding the risk factors for post-ERCP infections and the effectiveness of prevention and treatment methods, our work determined the type of ERCP surgery associated with the highest incidence of healthcare-associated infections (HAI), and investigated the pathogenic spectrum and antibiotic resistance of the infecting bacteria. Our study involved surveillance in one of the largest tertiary hospitals in China from 2012 to 2015 .

\section{Methods \\ Definitions}

1) Post-ERCP infection was defined as the HAI occurring on the day of operation or within 30 days post-surgery. The HAI criterion was consistent with that set by the Centers for Disease Control-National Healthcare Safety Network (CDC-NHSN) in the USA [6]. Infections observed for 30 days post-ERCP adhered to the Chinese criteria for post-surgery infections [7]. Infections definitely not caused by other operations unrelated to ERCP, within 30 days post-ERCP, were included.

2) Cholangitis was defined based on the Tokyo Guidelines 2007 [8] and the criteria included: 1 ) the presence of the following signs or symptoms after ERCP: fever $\left(>38.0^{\circ} \mathrm{C}\right)$, chill, abdominal pain, jaundice and liver biochemistry suggestive of biliary obstruction; 2) laboratory data indicative of the presence of inflammation and biliary obstruction; 3) imaging findings indicative of biliary obstruction, and 4) no evidence of acute cholangtitis in the week prior to the ERCP operation.

3) Bacteremia was defined as the presence of fever, chill and positive bacteria cultures from blood samples (excluding contamination, as previously described [9]) post-surgery, according to the definition of bloodstream infections from the CDC-NHSN [10]. 3.1-I) Secondary bacteremia was considered when the infection could definitely be sourced to one infected organ or site. For all cases of secondary bacteremia, the infection site was not recounted.
3.1-II) Secondary bacteremia following an infection at another site/not related to ERCP was excluded. 3.2-I) Transient primary bacteremia was counted at the infection site if positive bloodstream infections indicators (i.e., fever $\left(>38.0^{\circ} \mathrm{C}\right)$, chill) and positive blood culture results were observed.

3.2-II) Transient primary bacteremia was counted at the infection site only if positive bloodstream infections indicators (i.e., fever $\left(>38.0^{\circ} \mathrm{C}\right)$, chill), confirmed the clinical diagnosis, and negative blood culture results were observed.

\section{Post-ERCP infection data collection}

Post-ERCP infection data were collected at a tertiary hospital in Beijing, from 2012 to 2015. The hospital contained approximately 3800 beds, with 160 beds in the gastroenterology department. Each day, around two to four ERCP operations were performed in this hospital. Three senior clinical doctors, with more than 3 years of experience in ERCP operations, were in turn in charge of ERCP operations.

Real-time automatic hospital-wide surveillance of HAIs and outbreaks has been established in this hospital [11]. In brief, this system can automatically download microbiological reports, antibiotic usage, imaging reports, fever history and other information, and subsequently identify new HAIs in real time, and record and analyze the data. We used this system to collect postERCP infection data, including continuous fever (temperature $\geq 38.0{ }^{\circ} \mathrm{C}$ for $>2$ days), positive microbiological cultures and new antibiotic administration post-surgery. The system can develop post-ERCP HAI prewarning alerts. Subsequently, infection control practitioners work with doctors to confirm post-ERCP HAIs, as described in a previous study [11].

\section{Statistical analysis}

Differences in categorical variables were assessed using a Pearson $X[2]$ test or Fisher's exact test (when expected cell frequencies were $<5$ ). SPSS version 20.0 was used for all statistical analyses. A two-tailed $p$ value of $<0.05$ was considered to be statistically significant.

\section{Results \\ ERCP inpatients}

From 2012 to 2015, a total of 1743 ERCP operations on 1660 patients were successfully carried out in the hospital. Of these cases, 1034 (62.29\%) were male and 626 (37.71\%) were female. Patients aged from 10 to 98 years old, with a median age of 61 years old. The median length of stay was 6 days. Reasons for ERCP included bile duct calculi (452, 27.23\%), biliary pancreatic malignant tumors $(365,21.99 \%)$, obstructive jaundice $(313,18.86 \%)$, acute pancreatitis $(224,13.49 \%)$, 
cholangitis (157, 9.46\%), cholelithiasis or cholecystitis (78, 4.70\%) and other (71, 4.28\%).

\section{Post-ERCP infection}

Over the study period, a total of 132 HAIs occurred in 125 patients undergoing 1743 ERCP operations. The HAI prevalence was $7.57 \%(n=132 / 1743)$. The median time to develop a post-ERCP infection was 7 days. Seven patients died and the other 1653 patients were discharged.

ERCP postoperative infections occurred following different surgical procedures, with infection rates ranging from 3.58 to $13.51 \%$. The total number of HAIs and biliary tract infections that occurred following different types of operations are shown in Table 1. The three most prevalent post-ERCP infections were associated with bile duct or biliary stent implantation (13.51\%), bile duct stent removal and replacement (10.42\%) and bile duct stone removal operations (10.14\%). There were no differences between the rates of infection for therapeutic ERCP $(7.83 \%)$ and diagnostic ERCP $(4.51 \%)(p=0.165)$. The most prevalent HAI post-ERCP infections were biliary tract infections $(4.02 \%)$, followed by transient bacteremia $(1.14 \%)$, lower respiratory tract infections (0.91\%), upper respiratory tract infections (0.54\%), gastrointestinal infections $(0.42 \%)$, urinary tract infections $(0.18 \%)$ and others $(0.42 \%)$.

\section{Post-ERCP bacteremia}

A total of 62 bacteremia cases occurred post-ERCP, including 20 cases of transient and 42 cases of secondary bacteremia. All cases of bacteremia occurred within 14 days post-surgery. The majority of cases occurred on

Table 1 Prevalence of post-ERCP HAls and biliary tract infections for different types of ERCP operations

\begin{tabular}{|c|c|c|c|}
\hline Operations & $\begin{array}{l}\text { No. of } \\
\text { operations }\end{array}$ & $\begin{array}{l}\text { No. }(\%) \\
\text { of HAls }\end{array}$ & $\begin{array}{l}\text { No. (\%) of biliary } \\
\text { tract infections }\end{array}$ \\
\hline Diagnostic ERCP & 133 & $6(4.51)$ & $5(3.76)$ \\
\hline Diagnostic ERCP & 133 & $6(4.51)$ & $5(3.76)$ \\
\hline Therapeutic ERCP & 1610 & $126(7.83)$ & $65(4.03)$ \\
\hline $\begin{array}{l}\text { Lithotomy of duodenal } \\
\text { papilla }\end{array}$ & 727 & $26(3.58)$ & $7(0.96)$ \\
\hline Biliary stent implantation & 570 & $77(13.51)$ & $47(8.25)$ \\
\hline $\begin{array}{l}\text { Pancreatic duct stent } \\
\text { implantation }\end{array}$ & 137 & $7(5.11)$ & $0(0)$ \\
\hline Bile duct lithotomy & 69 & $7(10.14)$ & $4(5.80)$ \\
\hline $\begin{array}{l}\text { Bile duct stent extraction } \\
\text { and replacement }\end{array}$ & 48 & $5(10.42)$ & $3(6.25)$ \\
\hline Other therapeutic ERCP & 59 & $4(6.78)$ & $4(6.78)$ \\
\hline Total & 1743 & $132(7.57)$ & $70(4.02)$ \\
\hline
\end{tabular}

HAls included biliary tract infections, transient primary bacteremia, and respiratory tract infections

$E R C P$ endoscopic retrograde cholangiopancreatography, HAls healthcare-associated infections the day of surgery or 1 day post-surgery, which together accounted for $54.84 \%(34 / 62)$ of cases. All 20 cases of transient bacteremia occurred on the day of ERCP surgery and 1 day post-surgery. There were 42 cases of secondary bacteremia related to ERCP, including $39 \mathrm{sec}-$ ondary to biliary tract infections, one secondary to catheter-related bloodstream infections, one secondary to lower respiratory tract infections and one secondary to abdominal infections.

In addition, 58 pathogenic bacteria were cultivated from 62 patients. The majority $(60.34 \%)$ were gram negative bacteria (Table 2). The most prevalent isolates were 12 Enterococcus faecium and 13 Escherichia coli. Overall, $72.73 \%$ of E. coli and $100.00 \%$ of E. faecium isolates were resistant to ciprofloxacin. In addition, only $37.50 \%$ of the $E$. coli isolates were susceptible to ceftriaxone. Gram-negative E. coli isolates were $100.00 \%$ susceptible to imipenem. The imipenem resistance rate for non-fermenting bacteria (five Pseudomonas and four Acinetobacter baumannii) reached 80.00-100.00\%. Gram-positive E. faecium was $100.00 \%$ susceptible to vancomycin and linezolid (Table 3).

\section{Discussion}

To our knowledge, this is the first report on post-ERCP infections in Chinese tertiary hospitals. Previous studies have indicated that therapeutic ERCP endoscopic procedures are associated with the highest rates of bacteremia and other infectious complications [3, 12]. Our study had a clear definition for HAIs post-ERCP operations, which excluded all infections that occurred before the ERCP. Using this definition, the overall prevalence of

Table 2 The bacteriology of positive blood cultures post-ERCP

\begin{tabular}{ll}
\hline Organisms & No. (\%) of organisms \\
\hline Gram-positive organisms & $22 / 58(37.93 \%)$ \\
Enterococcus faecium & $12 / 58(20.69 \%)$ \\
Enterococcus faecalis & $1 / 58(1.72 \%)$ \\
Staphylococcus spec. & $4 / 58(6.90 \%)$ \\
Others & $5 / 58(8.62 \%)$ \\
Gram-negative organisms & $35 / 58(60.34 \%)$ \\
Escherichia coli & $11 / 58(18.97 \%)$ \\
Klebsiella pneumonia & $5 / 58(8.62 \%)$ \\
Pseudomonas aeruginosa & $5 / 58(8.62 \%)$ \\
Enterobacter cloacae & $4 / 58(6.90 \%)$ \\
Acinetobacter baumannii & $4 / 58(6.90 \%)$ \\
Stenotrophomonas maltophilia & $2 / 58(3.45 \%)$ \\
Aeromonas spec. & $2 / 58(3.45 \%)$ \\
Others & $2 / 58(3.45 \%)$ \\
Fungi & $1 / 58(1.72 \%)$ \\
Candida glabrata & $1 / 58(1.72 \%)$ \\
\hline
\end{tabular}


Table 3 Antibiotic susceptibilities of different blood culture isolates

\begin{tabular}{lllllll}
\hline Antibiotics & Enterococcus faecium & Escherichia coli & Klebsiella pneumonia & Pseudomonas aeruginosa & Acinetobacter baumannii & Enterobacter cloacae \\
\hline Aamikacin & - & $100 \%(11 / 11)$ & $100 \%(5 / 5)$ & $40 \%(2 / 5)$ & - & $100 \%(4 / 4)$ \\
Ampicillin & $8 \%(1 / 12)$ & $14 \%(1 / 7)$ & $0 \%(0 / 4)$ & $0 \%(0 / 5)$ & $0 \%(0 / 4)$ & $0 \%(0 / 2)$ \\
Aztreonam & - & $38 \%(3 / 8)$ & $100 \%(4 / 4)$ & $50 \%(2 / 4)$ & $0 \%(0 / 3)$ & $50 \%(1 / 2)$ \\
Nitrofurantoin & $33 \%(4 / 12)$ & $63 \%(5 / 8)$ & $50 \%(2 / 4)$ & $0 \%(0 / 3)$ & $0 \%(0 / 3)$ & $50 \%(1 / 2)$ \\
Ciprofloxacin & $0 \%(0 / 12)$ & $27 \%(3 / 11)$ & $80 \%(4 / 5)$ & $60 \%(3 / 5)$ & $0 \%(0 / 4)$ & $100 \%(4 / 4)$ \\
Gentamicin & $38 \%(3 / 8)$ & $75 \%(6 / 8)$ & $100 \%(4 / 4)$ & $33 \%(1 / 3)$ & $33 \%(1 / 3)$ & $100 \%(2 / 2)$ \\
Cefazolin & - & $0 \%(0 / 6)$ & $0 \%(0 / 4)$ & $0 \%(0 / 3)$ & $0 \%(0 / 3)$ & $0 \%(0 / 2)$ \\
Ceftazidime & - & $45 \%(5 / 11)$ & $80 \%(4 / 5)$ & $60 \%(3 / 5)$ & $0 \%(0 / 4)$ & $25 \%(1 / 4)$ \\
Ceftriaxone & - & $38 \%(3 / 8)$ & $75 \%(3 / 4)$ & $0 \%(0 / 3)$ & $0 \%(0 / 3)$ & $50 \%(1 / 2)$ \\
Cefepime & - & $64 \%(7 / 11)$ & $80 \%(4 / 5)$ & $40 \%(2 / 5)$ & - & $75 \%(3 / 4)$ \\
Vancomycin & $100 \%(12 / 12)$ & - & - & - & - & - \\
Linezolid & $100 \%(12 / 12)$ & - & - & $20 \%(1 / 5)$ & $0 \%(0 / 4)$ & $100 \%(4 / 4)$ \\
Imipenem & - & $100 \%(11 / 11)$ & $100 \%(5 / 5)$ & $60 \%(3 / 5)$ & $0 \%(0 / 3)$ & $100 \%(2 / 2)$ \\
Levofloxacin & $0 \%(0 / 8)$ & $29 \%(2 / 7)$ & $100 \%(4 / 4)$ & &
\end{tabular}

post-ERCP was $7.57 \%$. However, it must be considered that, for example, if cholangitis occurred post-ERCP whether it was a complication of ERCP or just a natural history of gallbladder stones. The prevalence of bacteremia post-ERCP was $3.56 \%(62 / 1743)$ in our study, which was far lower than in other studies from other countries. Both Thosani et al. [3] in the USA and Kullman et al. [11] in Sweden reported the incidence of bacteremia associated with ERCP to be as high as $27 \%$. The major reason for the high incidence might be that in these studies, two or three blood samples were obtained from each patient immediately before and after ERCP, to test the pathogenic spectrum. This active surveillance might increase the positive detection rate of bacteremia. In addition, these studies did not distinguish between primary and secondary bacteremia. Our study was based on daily medical work and only $17.73 \%$ (309/ 1743) of inpatients with suspected bacteremia provided samples for blood cultures.

In our study, the most prevalent infection post-ERCP was cholangitis (4.02\%; 70/1743). Ertugrul et al. [13] reported a similar result, that $3.3 \%(17 / 503)$ of patients developed cholangitis after ERCP. In addition, the risk of cholangitis was significantly higher in patients with biliary dilatation and biliary stent insertion. Our study indicated that surgery for biliary tract disease was associated with the highest risk of infection. Surgery on the biliary tract frequently uses a basket or balloon lithotomy and biliary stent implantation is difficult and exposes patients to a high risk of bile duct injury. Bacterial colonization in the gallbladder or bile duct increases the risk of biliary tract infection post-surgery.

Our analysis showed the predominance of gramnegative organisms (60.34\%) in the blood cultures, compared with gram-positive organisms (37.93\%). The most prevalent isolates were E. faecium and E. coli. Rerknimitr et al. [14] reported similar findings in patients undergoing ERCP, which indicated that Enterococcus spp. and E. coli were the most prevalent organisms isolated from bile cultures. In addition, Thosani et al. [3] reported that E. coli and Enterococcus spp. were the most prevalent causes of bacteremia post-ERCP.

Park et al. [15] confirmed that E. coli and Enterococcus spp. were the most prevalent organisms isolated from the blood cultures of biliary tract-infected patients. According to patient outcomes, prophylactic antibiotics are thought to be the most effective prevention against infections from gram-negative bacteria and Enterococcus spp. Several guidelines [16-19] have been published about antibiotic prophylaxis prior to ERCP. Most guidelines [17, 18], such as the American Society for Gastrointestinal Endoscopy and the British Society of Gastroenterology Endoscopy, advocate prophylaxis for patients with biliary obstruction, whereas the European Society of Gastrointestinal Endoscopy [19] recommends prophylaxis for every type of therapeutic ERCP. Antibiotic prophylaxis involves administering an antibiotic to combat biliary microorganisms, such as E. coli, Klebsiella pneumoniae and Enterococcus spp. Oral ciprofloxacin, intravenous piperacillin-tazobactam or gentamicin is recommended in the USA [20].

In China, the excessive long-term use of antibiotics by hospitals and the widespread addition of quinolones to animal feed has led to the emergence of significant levels of bacterial resistance to many antimicrobials [21, 22]. For example, bacteria of the Enterobacteriaceae (especially $E$. coli) are resistant to fluoroquinolones and Xiao et al. reported that about $65 \%$ of $E$. coli strains were 
resistant to fluoroquinolones between 2004 and 2005 [21]. In our study, only $27 \%$ of $E$. coli isolates were found to be susceptible to ciprofloxacin. This limits the use of fluoroquinolones as prophylactic drugs in China. The 2015 Chinese Clinical Application of Antibacterial Drugs guidelines [23] recommended second generation cephalosporin or ceftriaxone as the antibiotics of choice for prophylaxis for the pathogenic bacteria that can cause skin incision infections after ERCP. However, all gram-negative organisms in our study were resistant to cefazolin. Nearly $90 \%$ of inpatients were treated with metronidazole and ceftriaxone prophylactic antibiotics in the study hospital; however, the drug susceptibility results revealed that only $38 \%$ of $E$. coli isolates were susceptible to ceftriaxone. The high infection rate after ERCP and the prevalence of drug-resistant pathogens indicate that further studies are important. A cost benefit analysis is also necessary to determine whether the recommended guidelines should be modified.

This study has several limitations. The rate of bacteremia may have been underestimated, because Chinese doctors in general less frequently submit blood specimens for bacterial culture [24]. Some cases of bacteremia would have therefore gone undetected because of a lack of blood culture results. Another issue is that our study monitored all ERCP cases during hospitalization but post-discharge surveillance was not carried out at 30 days post-ERCP procedure. Postdischarge surveillance could be performed to identify HAIs that were not been reported and documented in our research.

\section{Conclusions}

Compared with HAIs in the whole hospital [24], postERCP infections generally exhibited a higher incidence $(7.6 \%$ vs. $4.1 \%)$ and different types of infections (cholangitis vs. respiratory tract infections). The detailed epidemiological data for HAIs after ERCP in China may improve our understanding of the severity of post-ERCP infections and potential prevention and treatment measures. Our study indicates that the incidence of postERCP infection is high and that drug resistance among the causative bacteria is common; thus, we suggest that the use of second generation cephalosporin or ceftriaxone as the antibiotic of choice for prophylaxis before ERCP may not be effective. Therefore, we recommend administering a high-grade antibiotic (i.e. piperacillintazobactam), which would target most biliary organisms, before ERCP.

\section{Abbreviations}

CDC-NHSN: Centers for Disease Control-National Healthcare Safety Network ERCP: Endoscopic retrograde cholangiopancreatography; HAl: Healthcareassociated infection

\section{Acknowledgements}

We thank all of our colleagues in the Gastroenterology Department for making this study possible. We wish to express our most sincere appreciation to Prof $\mathrm{Na}$ Jia who read the manuscript carefully and gave valuable advice.

\section{Funding}

This study was supported by grants from the China Special Grant for the Prevention and Control of Infectious Diseases (2013ZX10004 805-003,

2013ZX10004 217-002)

\section{Availability of data and materials}

The datasets generated during the current study are not publicly available, to avoid disclosure of the individual privacy of the patients. However, they are available from the corresponding author on reasonable request.

\section{Authors' contributions}

$\mathrm{MD}$ and JS collected the data, interpreted the results and wrote the manuscript. $\mathrm{BL}$ and $\mathrm{YX}$ collected and analyzed the data. $\mathrm{LC}$ and $\mathrm{YL}$ designed the study and revised the manuscript. All authors read and approved the final manuscript.

\section{Ethics approval and consent to participate}

This study was approved by the studied hospital institutional review board. For this type of study, formal consent is not required.

Consent for publication

Not applicable

Competing interests

The authors declare that they have no competing interests.

\section{Publisher's Note}

Springer Nature remains neutral with regard to jurisdictional claims in published maps and institutional affiliations.

Received: 5 September 2017 Accepted: 18 December 2017

Published online: 29 December 2017

\section{References}

1. Hu LH, Xin L, Liao Z, Pan J, Qian W, Wang LW, et al. ERCP development in the largest developing country: a national survey from China in 2013. Gastrointest Endosc. 2016;84(4):659-66.

2. Shorvon PJ, Eykyn SJ, Cotton PB. Gastrointestinal instrumentation, bacteremia, and endocarditis. Gut. 1983:24(11):1078-93.

3. Thosani N, Zubarik RS, Kochar R, Kothari S, Sardana N, Nguyen T, et al. Prospective evaluation of bacteremia rates and infectious complications among patients undergoing single-operator choledochoscopy during ERCP. Endoscopy. 2016:48(5):424-31.

4. Cotton PB, Connor P, Rawls E, Romagnuolo J. Infection after ERCP, and antibiotic prophylaxis: a sequential quality-improvement approach over 11 years. Gastrointest Endosc. 2008;67(3):471-5.

5. Testoni PA. Why the incidence of post-ERCP pancreatitis varies considerably? Factors affecting the diagnosis and the incidence of this complication. Journal of the Pancreas. 2002;3(6):195-201.

6. Identifying Healthcare-associated Infections (HAI) for NHSN Surveillance. https://www.cdc.gov/nhsn/pdfs/pscmanual/2psc_identifyinghais_ nhsncurrent.pdf. Accessed 30 Aug 2017

7. The Chinese Hospital infection diagnostic criteria. 2001. http://www.nhfpc gov.cn/yzygj/s3593/200804/e19e4448378643a09913ccf2a055c79d.shtml. Accessed 30 Aug 2017

8. Wada K, Takada T, Kawarada Y, Nimura Y, Miura F, Yoshida M, et al. Diagnostic criteria and severity assessment of acute cholangitis: Tokyo guidelines. J Hepato-Biliary-Pancreat Surg. 2007;14(1):52-8.

9. Hall KK, Lyman JA. Updated review of blood culture contamination. Clin Microbiol Rev. 2006;19(4):788-802.

10. Bloodstream Infection Event (Central Line-Associated Bloodstream Infection and Non-central line-associated Bloodstream Infection). http://www.cdc. gov/nhsn/pdfs/pscmanual/4psc-clabscurrent.pdf. Accessed 30 Aug 2017.

11. Du M, Xing Y, Suo J, Liu B, Jia N, Huo R, et al. Real-time automatic hospitalwide surveillance of NIs and outbreaks in a large Chinese tertiary hospital. BMC Med Inform Decis Mak. 2014;14:9. 
12. Kullman E, Borch $\mathrm{K}$, Lindström $\mathrm{E}$, Anséhn $\mathrm{S}$, Ihse I, Anderberg B. Bacteremia following diagnostic and therapeutic ERCP. Gastrointest Endosc. 1992;38(4):444-9.

13. Ertuğrul I, Yüksel I, Parlak E, Ciçek B, Ataseven H, Başar O, et al. Risk factors for endoscopic retrograde cholangiopancreatography-related cholangitis: a prospective study. Turk J Gastroenteral. 2009;20(2):116-21.

14. Rerknimitr R, Fogel EL, Kalayci C, Esber E, Lehman GA, Sherman S. Microbiology of bile in patients with cholangitis or cholestasis with and without plastic biliary endoprosthesis. Gastrointest Endosc. 2002;56(6):885-9.

15. Park JW, Lee JK, Lee KT, Lee KH, Sung YK, Kang Cl. How to interpret the bile culture results of patients with biliary tract infections. Clin Res Hepatol Gastroenterol. 2014;38(3):300-9.

16. Ceyssens C, Frans JM, Christiaens PS, Van Steenbergen W, Peetermans WE. Recommendations for antibiotic prophylaxis before ERCP: can we come to workable conclusions after review of the literature? Acta Clin Belg. 2006;61 (1):10-8.

17. Hirota WK, Petersen K, Baron TH, Goldstein JL, Jacobson BC, Leighton JA, et al. Guidelines for antibiotic prophylaxis for Gl endoscopy. Gastrointest Endosc. 2003;58(4):475-82.

18. Mani V, Cartwright K, Dooley J, Swarbrick E, Fairclough P, Oakley C. Antibiotic prophylaxis in gastrointestinal endoscopy: a report by a working Party for the Britisch Society of gastroenterology endoscopy committee. Endoscopy. 1997;29(2):114-9.

19. Rey JR, Budzynska A, Axon A, Kruse A, Nowak A. Guidelines of the European Society of Gastrointestinal Endoscopy (E.S.G.E.) antibiotic prophylaxis for gastrointestinal endoscopy. European Society of Gastrointestinal Endoscopy. Endoscopy. 1998;30(3):318-24.

20. Kovaleva J, Peters FT, van der Mei HC, Degener JE. Transmission of infection by flexible gastrointestinal endoscopy and bronchoscopy. Clin Microbiol Rev. 2013;26(2):231-54

21. Xiao Y, Li L. Legislation of clinical antibiotic use in China. Lancet Infect Dis. 2013;13(3):189-91.

22. Xiao YH, Wang J, Li Y. MOH National Antimicrobial Resistance Investigation net. Bacterial resistance surveillance in China: a report from Mohnarin 20042005. Eur J Clin Microbiol Infect Dis. 2008:27(8):697-708.

23. Chinese clinical application of antibacterial drugs guidelines 2015. http:// www.nhfpc.gov.cn/yzygj/s3593/201508/ c18e1014de6c45ed9f6f9d592b43db42.shtml.

24. Xie DS, Xiong W, Xiang LL, Fu XY, Yu YH, Liu L, et al. Point prevalence surveys of healthcare-associated infection in 13 hospitals in Hubei Province, China, 2007-2008. J Hosp Infect. 2010;76(2):150-5.

\section{Submit your next manuscript to BioMed Central and we will help you at every step:}

- We accept pre-submission inquiries

- Our selector tool helps you to find the most relevant journal

- We provide round the clock customer support

- Convenient online submission

- Thorough peer review

- Inclusion in PubMed and all major indexing services

- Maximum visibility for your research

Submit your manuscript at www.biomedcentral.com/submit 